\title{
INTOLERÂNCIA À LACTOSE E SUAS CONSEQUÊNCIAS NA SAÚDE DA MULHER
}

\section{INTOLERANCE TO LACTOSE AND ITS CONSEQUENCES IN WOMEN'S HEALTH}

\section{Geny Pereira Filha;}

Bacharel em Farmácia. Alfa - Faculdade de Almenara, Almenara-MG

Brasil.

\section{Laise Fernandes Rodrigues}

Bacharel em Farmácia. Alfa - Faculdade de Almenara, Almenara-MG

Brasil.

\section{Leonardo Henrique Guimarães Reis ;}

Especialista. Alfa - Faculdade de Almenara, Almenara-MG Brasil.

Viviane Amaral Toledo Coelho

Doutora.vivianeatc@yahoo.com.br. Almenara-MG

Patrícia Alves Cardoso

Mestre. Alfa - Faculdade de Almenara, Almenara-MG, Brasil.

\section{RESUMO}

Objetivo: Compreender os impactos da intolerância à lactose na saúde da mulher. Metodologia: O estudo foi realizado por meio de levantamento bibliográfico, descritivo, cujo propósito foi reunir conhecimentos sobre os impactos da intolerância a lactose na saúde da mulher. A pesquisa bibliográfica foi realizada em artigos científicos publicados entre os anos 2012 a 2019. O trabalho teve a duração de 5 meses, englobando de agosto/2019 a dezembro/2019. Resultados: As mulheres com IL devem ter a reposição adequada do cálcio $(\mathrm{Ca})$, por meio de outras fontes e/ou suplementação 
deste componente para atender aos níveis diários recomendados. A vitamina $\mathrm{D}$ pode ser encontrada sob as formas de ergocalciferol ou vitamina D2 e de colecalciferol ou vitamina D3, obtida através de leveduras e plantas produzidas para uso comercial. Conclusões: Apresentou-se alternativas alimentares e medicamentosas. No tocante as alternativas alimentares existem no mercado produtos lácteos com zero por cento de lactose. Dentre as medidas farmacológicas, para as mulheres, existe a terapia de reposição enzimática com a lactase exógena, além disso possuem as possibilidades de ingestão do cálcio e da vitamina D através de suplementação com uso comercial.

Palavras-chaves. Intolerância a lactose. Mulher. Medicamentos. Cálcio. Osteoporose.

\section{ABSTRACT}

Objective: To understand the impacts of lactose intolerance on women's health. Methodology: The study was conducted through a descriptive bibliographic survey, whose purpose was to gather knowledge about the impacts of lactose intolerance on women's health. The bibliographic research was performed in scientific articles published between the years 2012 to 2019. The work lasted 5 months, ranging from August / 2019 to December / 2019. Results: Women with IL should have adequate Ca replacement through other sources of calcium and / or supplementation of this component to meet recommended daily levels. Vitamin D can be found in the form of ergocalciferol or vitamin D2 and cholecalciferol or vitamin D3 obtained from yeast and plants produced for commercial use. Conclusions: Food and drug alternatives were presented. With regard to dietary alternatives there are dairy products with zero percent lactose on the market. Among the pharmacological measures, for women, there is enzyme replacement therapy with exogenous lactase, besides having the possibilities of calcium and vitamin D intake through supplementation with commercial use.

Keywords. Lactose intolerance. Woman. Medicines. Calcium. Osteoporosis. 


\section{INTRODUÇÃO}

O leite é definido como produto proveniente de uma ordenha completa e ininterrupta, em condições de higiene, de vacas sadias, bem alimentadas e descansadas (BRASIL, 2011). É um alimento consumido no mundo todo, utilizado desde os primórdios da humanidade para alimentação dos mamíferos. (SALOMÃO et al., 2012; BATISTA et al., 2018; MATHIÚS et al., 2016).

Por ser considerado como a fonte primária para obtenção do cálcio, o consumo diário do leite é muito importante, pois possui em média $120 \mathrm{mg}$ de cálcio por $100 \mathrm{~g}$ do alimento. O cálcio é um mineral fundamental para a manutenção das funções do organismo, como na formação de dentes e ossos, mitose, contração muscular, manutenção da matriz óssea, transporte na membrana celular, transmissão das sinapses e impulsos nervosos, coagulação do sangue, entre outros. (ZYCHAR; OLIVEIRA, 2017).

Também presente entre os componentes do leite encontra-se a lactose, principal carboidrato encontrado neste alimento. A lactose é um dissacarídeo constituído por glicose e galactose. No entanto, a capacidade de digerir a lactose presente no leite necessita da ação da enzima lactase (BAUERMANN; SANTOS, 2013; SANTOS et al., 2014). A má digestão da lactose é evidenciada quando a quantidade de lactose ingerida não é totalmente digerida e absorvida, devido ao declínio na expressão da atividade da lactase na idade adulta (lactase não persistente ou deficiente). Quando a quantidade de lactose ingerida é maior do que a quantidade suportada pelo organismo, são evidenciados os sintomas de intolerância à lactose (IL) (PEREIRA et al., 2012).

A IL é caracterizada pela redução ou ausência da produção da enzima lactase que tem a função de hidrolisar a lactose em glicose e galactose para serem absorvidas pelo intestino delgado. Alguns indivíduos quando são diagnosticados com a intolerância à lactose excluem ou reduzem o consumo de leite e derivados, como consequência poderão ter uma menor ingestão de cálcio e outros nutrientes fornecidos pelo leite como 
fósforo, potássio, magnésio, vitamina $\mathrm{D}$ e riboflavina. Além disso, a redução ou a não ingestão de produtos lácteos pode ocasionar uma diminuição da densidade mineral óssea com possibilidades de fraturas, elevar o risco de osteoporose, hipertensão e câncer de cólon (DANTAS; VERRUCK; PRUDENCIO, 2019; HODGES et al., 2019).

Atualmente a comercialização e o consumo de produtos lácteos com baixo teor ou isentos de lactose aumentou consideravelmente, favorecendo o público que tem a opção de continuar a se beneficiar dos nutrientes essenciais presentes nestes produtos, sem, portanto, sentir os sintomas indesejados da IL como flatulência, inchaço e dor abdominal, náusea, aumento da motilidade intestinal, entre outros (MATHIÚS et al.,2016; DI COSTANZOA; CANAN, 2018; DANTAS; VERRUCK; PRUDENCIO, 2019).

As mulheres com IL destacam-se como um dos grupos favorecidos com os produtos com baixo teor ou isentos de lactose, pois poderão continuar consumindo produtos derivados do leite contribuindo para a redução dos riscos, principalmente, de osteoporose. O aumento da idade das mulheres e as alterações hormonais relacionados a menopausa tendem a reduzir a densidade mineral óssea e aumentar o risco de osteoporose (SALOMÃO et al., 2012; PEREIRA et al., 2012). A alimentação saudável rica em produtos que forneçam uma maior biodisponibilidade de cálcio minimiza o risco de doenças ligadas à estrutura óssea, além de reduzir a necessidade de consumo de medicamentos para reposição de cálcio.

Assim, em função da existência da intolerância à lactose, muitas mulheres não consomem nenhum tipo de lácteo. Sendo assim, é necessário viabilizar alternativas de alimentos e de medicamentos para que a saúde da mulher não seja comprometida devido à intolerância à lactose. Desta forma, este trabalho é justificável e relevante, uma vez que as mulheres com a idade avançada já possuem alterações hormonais proveniente da menopausa o que compromete, em muitos casos, à absorção de cálcio implicando na possibilidade do desenvolvimento de osteoporose. 
Desta forma, o trabalho tem como objetivo geral compreender os impactos da intolerância à lactose na saúde da mulher. E como objetivos específicos verificar os impactos na saúde da mulher proveniente da restrição do consumo produtos lácteos devido à intolerância a lactose e apresentar alternativas de alimentos e medicamentos para mulheres com intolerância a essa patologia.

\section{METODOLOGIA}

O estudo foi realizado por meio de levantamento bibliográfico, descritivo, cujo propósito foi reunir conhecimentos sobre os impactos da intolerância a lactose na saúde da mulher. Fez-se de uma pesquisa qualitativa. A pesquisa bibliográfica foi realizada em artigos científicos publicados entre os anos 2012 a 2019, disponibilizados na base de dados Portal de Periódicos Capes, Biblioteca Virtual em Saúde Brasil (BVS) e Scientific Electronic Library Online (Scielo). Os descritores utilizados para a busca dos artigos nas bases de dados: "intolerância à lactose" AND "saúde"; "intolerância à lactose" AND "mulheres".

Os artigos foram avaliados por dois revisores que realizaram as triagens por meio da leitura dos títulos e resumos. Os artigos selecionados após a triagem foram lidos na integralidade. Foram utilizados como critério de inclusão artigos em português e inglês publicados na íntegra e excluídos os artigos em duplicidade e aqueles que não abordaram diretamente a relação da saúde da mulher com consumo de produtos lácteos.

De posse dos dados bibliográficos compilados os mesmos foram agrupados por conteúdos de relevância e discutidos dentro da literatura científica de forma a responder os objetivos propostos no presente artigo.

\section{ASPECTOS GERAIS DA LACTOSE}


A lactose é um dissacarídeo formado por uma D-glicose e uma D-galactose que se ligam por uma ligação $\beta$-1,4-glicosídica. É encontrada sob duas formas isoméricas cristalinas, sendo $\alpha$ hidratada e $\beta$ anidra (Figura 1) (PEREIRA et al., 2012; ZYCHAR; OLIVEIRA, 2017).

Figura 1: Estrutura química dos isômeros da lactose

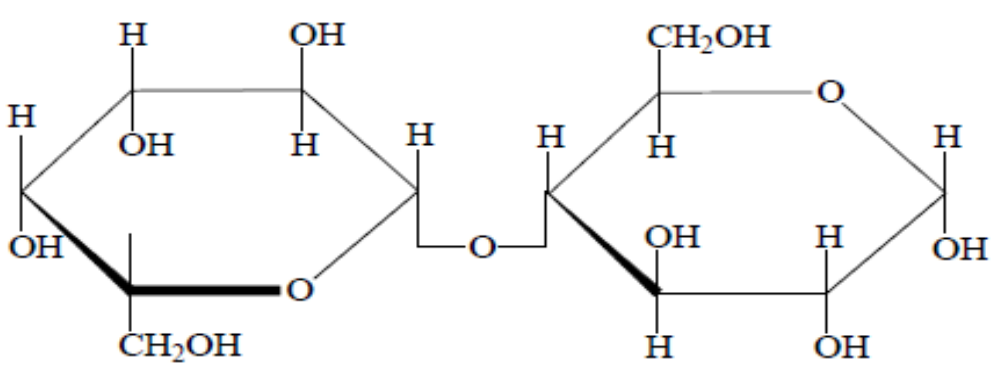

$\alpha$ lactose

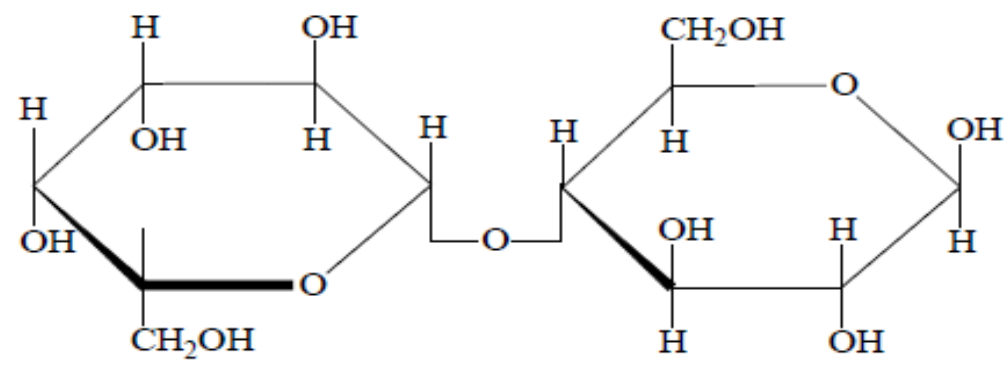

$\beta$ lactose

Fonte: Adaptado Santos et al., $(2014$, p. 2)

A lactose é um importante carboidrato que serve como fonte de energia, sua hidrólise ocorre na mucosa do intestino delgado pela ação da enzima lactase, também denominada de $\beta$-galactosidase. A glicose e galactose são os monossacarídeos facilmente solúveis e digeríveis provenientes da hidrólise da lactose (BAUERMANN; SANTOS 2013). Com o passar dos anos, é comum nos seres humanos ocorrer a 
diminuição na capacidade de absorção da lactose no intestino, levando com isso a uma má-absorção da lactose (SALOMÃO et al., 2012).

A lactose desempenha alguns papéis no organismo, conforme destaco por Zychar e Oliveira (2017, p. 38) atua como: fonte de energia, constituinte dos cerebrosídios, presentes na massa cerebral e mielina nervosa, atua também na retenção de cálcio e magnésio no organismo, além de prolongar a ação da vitamina $\mathrm{D}$ e inibir o desenvolvimento de bactérias putrefativas e patogênicas através da produção de ácido lático e diminuição do pH pela microflora. Assim, desempenha importantes papéis na saúde do organismo humano, como fonte de energia, retenção de cálcio e magnésio e vitamina D.

\section{ORIGENS DA INTOLERÂNCIA À LACTOSE}

Uma das razões da má-absorção da lactose está ligada a hipolactasia, que é a diminuição na quantidade da enzima lactase na mucosa do intestino delgado, o que ocasiona os sintomas comuns provenientes da intolerância à lactose (SANTOS et al., 2014). Os indivíduos com intolerância à lactose não têm a capacidade de digerir a lactose (BATISTA et al., 2018). A lactose que não é absorvida na digestão fica no intestino delgado, gerando no lúmen intestinal um aumento da pressão osmótica, levando a retenção de água e aumento do trânsito intestinal, ocasionando fezes amolecidas e até mesmo diarreia. Outros sintomas também podem aparecer como: flatulência, inchaço e dor abdominal, náusea, aumento da motilidade intestinal, entre outros (MATHIÚS et al., 2016; DI COSTANZO; CANAN, 2018).

Devido aos diferentes fatores que levam a deficiência da enzima lactase a IL pode ser classificada em três tipos: congênita, primária e secundária. A IL congênita é considerada uma condição autossômica recessiva grave, onde a atividade enzimática da lactase jejunal é ausente desde o nascimento e por toda a vida do indivíduo. É um 
problema raro e genético. O recém-nascido apresenta diarreia líquida ao receber o leito materno ou substitutos que contenham lactose. Caso não seja diagnosticada precocemente pode levar ao óbito em função da desidratação profunda (BATISTA et al., 2018).

Já a IL primária é uma condição autossômica recessiva, proveniente de uma alteração na regulação do gene da lactase. Nesse caso, existe uma redução na quantidade da lactase no organismo. É uma condição que surge já na primeira infância e perdura até a fase adulta, dificultando a absorção da lactose. Esse é o tipo de IL mais comum que acomete a população (BATISTA et al., 2018; DANTAS; VERRUCK; PRUDENCIO, 2019).

E por fim, a IL secundária que é uma condição transitória e reversível, que ocorre em função de uma lesão no intestino em decorrência de infecções, inflamação intestinal crônica, doença celíaca, doença de Crohn, bem como alterações na mucosa intestinal induzida por radiação/quimioterapia, entre outras (DI COSTANZOA; CANAN, 2018).

É necessário o diagnóstico adequado da IL, uma vez que existe equívoco por parte de alguns profissionais em diferenciar os termos alergia e intolerância. A intolerância alimentar é uma reposta do organismo a um alimento ou aditivo, sem a ocorrência de intervenções imunológicas. A alergia, ao contrário da intolerância, existe o processo de intervenção do sistema imunológico do indivíduo por meio de mecanismos de defesa contra antígenos, originando sinais e sintomas logo após a ingestão do alimento, como diarreia, urticária, náuseas, prurido, dor abdominal, constipação intestinal, broncoespasmos, entre outros (PEREIRA et al., 2012).

Existem métodos para realização do diagnóstico, sendo os mais comuns o teste de tolerância à lactose, teste respiratório do hidrogênio expirado, biopsia e genético (PEREIRA et al., 2012; DI COSTANZOA, CANAN, 2018). 


\section{A INTOLERÂNCIA A LACTOSE E SEUS IMPACTOS NA SAÚDE DA} MULHER

As mulheres com idade entre 40 a 65 anos passam por um período de transição do ciclo reprodutivo o chamado climatério. Nesta fase ocorrem mudanças de ordens biopsicossociais, afetivas, ocupacionais, sexuais, familiares, entre outras. No climatério, na fase da menopausa ocorre a interrupção permanente da menstruação. A menopausa ocorre por volta dos 48 a 50 anos de idade, e é um processo de transição na vida da mulher devido à ocorrência de um desequilíbrio hormonal. Os ovários não produzem mais óvulos, assim, cessa a menstruação e o organismo produz reduzida quantidade de estrogênios e progesterona. Pode acontecer antes que dos 40 anos ocorrer à interrupção precoce da menstruação, neste caso dá-se o nome de falência ovariana precoce (BRASIL, 2017 b; DIAS, 2012).

As mudanças fisiológicas no organismo provenientes das alterações hormonais, acabam tornando as mulheres mais propensas ao surgimento de algumas doenças e distúrbios, como doenças cardíacas, fadiga, depressão e osteoporose, entre outros (FERREIRA, et al., 2013). Nesta fase da vida da mulher é necessário a ingestão recomendada de micro e macro nutrientes para manter a saúde e prevenir doenças decorrentes da carência destes nutrientes, como, por exemplo, carência de $\mathrm{Ca}$ e a vitamina D. As deficiências nutricionais do cálcio e da vitamina D nas mulheres na pósmenopausa trazem consequências negativas sobre a matriz óssea aumentando o risco de fraturas osteoporóticas (BRINGEL et al., 2014).

O Ca é um importante micronutriente para o organismo humano, sendo considerado um regulador do metabolismo ósseo, importante no crescimento e obtenção do pico de massa óssea. O leite e seus derivados fornecem mais de $40 \%$ de $\mathrm{Ca}$ em sua composição. Outras fontes alimentares como peixes e vegetais verdes também são ricas em cálcio (BRINGEL et al., 2014; LEÃO; CARDOSO, 2014; HODGES, et al., 2019). 
O Ca é um nutriente essencial para os processos biológicos como na contração muscular, formação dos dentes, formação e manutenção da matriz óssea, transmissão de impulsos sinápticos, mitose, atividades enzimáticas, secreção de hormônios e coagulação do sangue. É o micronutriente mais abundante no corpo humano, sendo que 99\% encontram-se nos ossos e dentes e 1\% no sangue, nas células dos tecidos moles e no espaço extracelular. A quantidade de cálcio ideal ingerida produz uma elevação no pico da massa óssea, recomendado sua ingestão adequada em crianças, adolescentes e adultos, para que minimize a perda na velhice (SANTOS et al., 2014).

Bringel et al. (2014, p. 354) conceituam a osteoporose como: "um distúrbio osteometabólico, caracterizado pela perda de massa óssea e desarranjo da microarquitetura do tecido ósseo". A osteoporose é uma doença que ocorre em função da fragilidade da matriz óssea, favorecendo a ocorrências de fraturas por baixo impacto. A osteoporose está ligada a alguns fatores de risco na pós-menopausa, como baixa ingestão de cálcio, idade, etnia, baixa densidade mineral óssea - DMO, entre outras que contribuem para o agravamento da doença (RADOMINSKI, et al., 2017).

As mulheres que não consomem leite e seus derivados, em função da existência da intolerância à lactose, estão sujeitas a uma menor ingestão de cálcio e demais nutrientes fornecidos pelo leite (SANTOS et al., 2014; DANTAS; VERRUCK; PRUDENCIO, 2019).

Para atender à grande quantidade da população que sofrem com IL, atualmente já existem alternativas alimentares e até mesmo medicamentosas que auxiliam na manutenção das quantidades de micronutrientes fundamentais para promoção e preservação da saúde (DANTAS; VERRUCK; PRUDENCIO, 2019).

\section{RESULTADOS E DISCUSSÃO}


Na maioria dos casos, o diagnóstico da intolerância à lactose leva as mulheres à exclusão de sua alimentação os produtos lácteos. A completa exclusão dos lácteos da dieta sem um acompanhamento profissional leva, normalmente, ao comprometimento da absorção das quantidades necessárias de cálcio, vitaminas e outros minerais necessários para a manutenção do organismo da população feminina (MATHIÚS et al., 2016).

Para atender à grande quantidade da população de mulheres que sofrem com IL, atualmente já existem alternativas alimentares e até mesmo medicamentosas que auxiliam na manutenção das quantidades de micronutrientes fundamentais para promoção e preservação da saúde (DANTAS; VERRUCK; PRUDENCIO, 2019).

Para as mulheres que apresentam má absorção da lactose, já existe no mercado atual produtos lácteos com zero por cento de lactose - leites, leites fermentados, queijos, leite condensado, iogurtes, doces de leite. Os alimentos lácteos fermentados são indicados para a população feminina com má digestão da lactose, uma vez que a lactase dos microrganismos inseridos no processo de fabricação possibilita a hidrólise de parte da lactose contida nos produtos (PEREIRA, et al., 2012).

Através do leite sem lactose, em destaque, o produto pasteurizado, é possível desenvolver também os derivados do leite sem a lactose, existindo, portanto, os derivados lácteos isentos de lactose ("lac-free"), que são os produtos conhecidos como "zero lactose". Este processo do leite sem lactose é possível através da remoção da lactose pelo método de separação cromatográfico para eliminar a lactose total do alimento (DANTAS; VERRUCK; PRUDENCIO, 2019).

No Brasil, existem as resoluções RDC n 135/2017 e n 136/2017 da Agência Nacional de Vigilância Sanitária (ANVISA) que regulamentam as regras para os rótulos dos produtos, informando se possuem ou não lactose em sua composição. Segundo a resolução RDC n⿳亠口冋 136/2017, qualquer alimento que contenha quantidade acima de $0,1 \%$ de lactose deve conter a expressão "Contém lactose" no rótulo (BRASIL, 2017 b). 
Já a resolução RDC n 136/2017 diferencia alimentos com baixo teor de lactose dos isentos de lactose ou zero lactose, estipulando os dizeres que devem conter no rótulo dos produtos de acordo com as quantidades. Os alimentos que possuem quantidade abaixo de $100 \mathrm{mg} / 100 \mathrm{~g}$ ou $\mathrm{ml}$ de lactose enquadram nos dizeres "zero lactose, isento de lactose, $0 \%$ lactose, sem lactose ou não contém lactose”. Já aqueles que contém de $100 \mathrm{mg}$ até $1 \mathrm{~g} / 100 \mathrm{~g}$ ou $\mathrm{ml}$ devem ter escrito: "baixo teor de lactose ou baixo em lactose". Os alimentos com quantidade igual ou acima de $100 \mathrm{mg} / 100 \mathrm{~g}$ ou $\mathrm{ml}$ devem ter escrito "contém lactose".

As alternativas de medidas farmacológicas para as mulheres, pode ser realizada através da terapia de reposição enzimática com a lactase exógena, que pode ser obtida através de fungos e levedura, sendo comercializado como um alimento funcional e podem ser encontradas em comprimidos e na forma líquida. A terapia de reposição enzimática através da ingestão da lactase exógena foi recentemente aprovada pela ANVISA para comercialização. A recomendação para ingestão dos produtos com a lactase exógena, em comprimidos, é antes de consumir produtos lácteos, reduzindo assim as restrições alimentares (PEREIRA et al., 2012; FRANCESCONI et al., 2016; ZYCHAR; OLIVEIRA, 2017).

Como alternativas existe ainda, a possibilidade da ingestão adequada do $\mathrm{Ca}$ e Vitamina D, através de outras fontes de alimentos, não apenas no Leite e seus derivados, mas também através de alimentos fortificados com $\mathrm{Ca}$ e na forma de suplementação nutricional, pois contribui para a diminuiu da perda de DMO e redução dos danos em função da senilidade nas mulheres.

O Ca que tem seu armazenamento primário nos ossos é um componente indispensável a saúde das mulheres, sendo importante para o desenvolvimento e manutenção do sistema esquelético, como regulador metabólico e nos processos de excitação dos músculos. A recomendação diária de cálcio por dia é descrita na Tabela 1. 
Tabela 1 - Recomendação de ingestão diária de cálcio (mg/dia), de acordo com a faixa etária em Mulheres

\begin{tabular}{ll}
\hline Faixa etária & Recomendação (mg/dia) \\
\hline $19-50$ anos & $1000 \mathrm{mg} / \mathrm{dia}$ \\
Acima de 50 anos & $1200 \mathrm{mg} / \mathrm{dia}$ \\
\end{tabular}

Fonte: Barbosa; Andreazzi adaptado (2012, p. 84)

Assim, as mulheres com IL devem ter a reposição adequada do $\mathrm{Ca}$, por meio de outras fontes de cálcio e/ou suplementação deste componente para atender aos níveis diários recomendados (SILVA, 2017).

A Vitamina D atua na regulação da homeostase do cálcio e do metabolismo ósseo e sua deficiência pode resultar na má reabsorção óssea e perda da massa óssea e consequentemente no desenvolvimento de osteopenia e osteoporose, além da fraqueza muscular que pode levar a ocorrência de quedas nas mulheres (MAEDA et al., 2014). Na Tabela 2 é possível verificar a ingestão recomendada de Vitamina D de cálcio em mulheres por faixa etária.

Tabela 2 - Ingestão recomendada de Vitamina D (UI/dia), segundo a faixa etária em Mulheres

\begin{tabular}{ll}
\hline Faixa etária & Ingestão (UI/dia) \\
\hline $1-50$ anos & $200 \mathrm{UI} /$ dia \\
$51-70$ anos & $400 \mathrm{UI} /$ dia \\
Acima de 70 anos & $600 \mathrm{UI} /$ dia
\end{tabular}

Fonte: Barbosa; Andreazzi adaptado (2012, p. 84) 
Quando já diagnosticado o quadro de osteoporose nas Mulheres, é recomendado a suplementação dos níveis de Vitamina D conforme Tabela 3.

Tabela 3 - Vitamina D para Mulheres com osteoporose.

\begin{tabular}{lll}
\hline Níveis/valores & Níveis & Recomendação de suplementação \\
\hline$<20$ a $29 \mathrm{ng} / \mathrm{Ml}$ & Insuficiente & $7.000 \mathrm{UI} /$ dia ou $50.000 \mathrm{UI} / \mathrm{semana}$ por oito semanas \\
30 e $100 \mathrm{ng} / \mathrm{mL}$ & Suficiente & Não há necessidade
\end{tabular}

Fonte: Maeda et al., adaptado (2014)

A vitamina D pode ser encontrada sob as formas de ergocalciferol ou vitamina D2 e de colecalciferol ou vitamina D3, obtida através de leveduras e plantas produzidas para uso comercial (BRINGEL, et al., 2014; MAEDA et al., 2014).

\section{CONSIDERAÇÕES FINAIS}

A intolerância a lactose acomete muitas mulheres e contribui para o surgimento de algumas patologias em função da má absorção de nutrientes oriundos dos produtos lácteos.

Dentre os micronutrientes necessários para saúde da mulher tem-se o Cálcio e a Vitamina D. A ausência e/ou deficiência afeta principalmente no metabolismo ósseo contribuindo para a osteoporose, osteopenia e fraturas. 
Desta forma, a não ingestão dos produtos lácteos em função da intolerância a lactose devem ser supridas por outras fontes alternativas.

Neste trabalho, apresentou-se alternativas alimentares e medicamentosas. No tocante as alternativas alimentares existem no mercado produtos lácteos com zero por cento de lactose. Esta produção é regulamentada pela Agência Nacional de Vigilância Sanitária.

Dentre as medidas farmacológicas, para as mulheres, existe a terapia de reposição enzimática com a lactase exógena, recentemente aprovada pela ANVISA.

Além disso possuem as possibilidades de ingestão do cálcio e da vitamina D através de suplementação com uso comercial.

\section{REFERÊNCIAS BIBLIOGRÁFICAS}

BARBOSA, C. R. ANDREAZZI, M. A. Intolerância à lactose e suas consequências no metabolismo do cálcio. Revista Saúde e Pesquisa, v. 4, n. 1, p. 81-86, jan. / abr. 2012.

BATISTA, R. A. B. et al. Lactose em alimentos industrializados: avaliação da disponibilidade da informação de quantidade. Ciência \& Saúde Coletiva, v. 23, n. 12, p. 4119-4128, 2018.

BAUERMANN, A. SANTOS, Z. A. Conhecimento sobre intolerância à lactose entre nutricionistas. Scientia Medica, Porto Alegre, v. 23, n. 1, p. 22-27, 2013.

BRASIL. Instrução Normativa n. 62, de 29 de dezembro de 2011. Regulamento Técnico de Produção, Identidade e Qualidade do Leite tipo A. O Regulamento Técnico de Identidade e Qualidade de Leite Cru Refrigerado. Brasília: Ministério da Agricultura, Pecuária e Abastecimento, 2011.

BRASIL. Resolução - RDC n $^{\circ}$ 136, de 8 de fevereiro de 2017. Estabelece os requisitos para declaração obrigatória da presença de lactose nos rótulos dos alimentos. Brasília, 2017. a 
BRASIL. Resolução - RDC no 135, de 8 de fevereiro de 2017. Altera a Portaria SVS/MS n. 29, de 13 de janeiro de 1998, que aprova o regulamento técnico referente a alimentos para fins especiais, para dispor sobre os alimentos para dietas com restrição de lactose. Brasília, 2017. b

BRINGEL, A. L. et al. Suplementação nutricional de cálcio e vitamina D para a saúde óssea e prevenção de fraturas osteoporóticas. Revista Brasileira de Ciências da Saúde. v. 18, n. 4, p. 353-358, 2014.

DANTAS, A. VERRUCK, S. PRUDENCIO, E. S. Ciência e tecnologia de leite e produtos lácteos sem lactose. Ponta Grossa: Atena Editora, 2019. 74 p.

DIAS, I. M. Menopausa. Revista Brasileira Odontologia, v. 69, n. 1, p. 6-7, 2012.

DI COSTANZOA, M. CANAN, R. B. Lactose Intolerance: Common Misunderstandings. Anais of Nutrition \& Metabolism, v. 73, n. 4, p. 30-37, 2018.

FERREIRA, V. N. et al. Menopausa: marco biopsicossocial o envelhecimento feminino.

Psicologia \& Sociedade, v. 25, n. 2, p. 410-419, 2013.

FRANCESCONI, C. F. M. et al. Administração oral de lactase exógena em comprimidos em pacientes portadores de intolerância à lactose devido à hipolactasia primária. Arquivo Gastrenterologia, v. 53, n. 4, p. 228-234, 2016.

HODGES, J. K. et al. Lactose intolerance and bone health: the challenge of ensuring adequate calcium intake. Nutrientes Review, v. 11, n. 4, p. 1-17, 2019.

LEAO, L. S. CARDOSO, F. S. Efeitos do consumo de cálcio na composição corporal e perda de peso em adultos. Revista Brasileira de Ciências da Saúde, v. 12, n. 40, p. 69-73, abr. / jun. 2014.

MAEDA, S. S. et al. Recomendações para o diagnóstico e tratamento da hipovitaminose D. Arquivo Brasileiro Endocrinologia Metabólica, v. 58, n. 5, p. 411-433, 2014. 
MATHIÚS, L. A. et al. Aspectos atuais da intolerância à lactose. Revista Odontológica de Araçatuba, v. 37, n. 1, p. 46-52, jan. / abr. 2016.

PEREIRA, M. C. S. et al. Lácteos com baixo teor de lactose: uma necessidade para portadores de má digestão da lactose e um nicho de mercado. Revista do Instituto de Laticínios Cândido Tostes, v. 67, n. 389, p. 57-65, nov. / dez. 2012.

RADOMINSKI, S. C. et al. Diretrizes brasileiras para o diagnóstico e tratamento da osteoporose em mulheres na pós-menopausa. Revista Brasileira Reumatologia, v. 57, n. 2, p. 452-644, 2017.

SALOMÃO, N. A. et al. Ingestão de cálcio e densidade mineral óssea em mulheres adultas intolerantes à lactose. Revista Nutrição, Campinas, v. 25, n. 5, p. 587-595, set. / out. 2012.

SANTOS, F. F. P. et al. Intolerância à lactose e as consequências no metabolismo do cálcio. Revista Interfaces: Saúde, Humanas e Tecnologia, v. 2, n. 2, edição especial, jun. 2014.

SILVA, C.M.E. A intolerância à lactose e as consequências na absorção do cálcio. Revista Eletrônica Atualiza Saúde, Salvador, v. 6, n. 6, p. 29-35, jul. / dez. 2017.

ZYCHAR, B. C. OLIVEIRA, B. A. Fatores desencadeantes da intolerância á lactose: metabolismo enzimático, diagnóstico e tratamento. Atas de Ciências da Saúde, São Paulo, v. 15, n. 1, p. 35-46, jan. / mar. 2017. 
SAÚdista
SAL VALES 